Pacific Journal of Mathematics

STRONGLY RECURRENT TRANSFORMATIONS 


\title{
STRONGLY RECURRENT TRANSFORMATIONS
}

\author{
ARshag HaJian
}

Let $(X, \mathscr{B}, m)$ be a finite or $\sigma$-finite and non-atomic measure space. A set $\mathrm{B}$ is said to be measurable if it is a member of $\mathscr{B}$. Two measures on $\mathscr{B}$, finite or $\sigma$-finite (one may be finite and the other $\sigma$-finite), are said to be equivalent if they have the same null sets. In this paper we consider a one-to-one, nonsingular, measurable transformation $\phi$ of $X$ onto itself. By a nonsingular transformation $\phi$ we mean $m(\phi B)=m\left(\phi^{-1} B\right)=0$ for every measurable set $B$ with $m(B)=0$, and by a measurable transformation $\phi$ we mean $\phi B \in \mathscr{B}$ and $\phi^{-1} B \in \mathscr{B}$ for every $B \in \mathscr{B}$. We shall say that the transformation $\phi$ is measure preserving (with respect to a measure $\mu$ ) or equivalently, $\mu$ is an invariant measure (with respect to the transformation $\phi$ ) if $\mu(\phi B)=\mu\left(\phi^{-1} B\right)=\mu(B)$ for every measurable set $B$.

A recurrent transformation is a common notion in ergodic theory. This is a measurable transformation $\phi$ defined on a finite or $\sigma$-finite measure space $(X, \mathscr{B}, m)$ with the following property: if $A$ is a measurable set of positive measure, then for almost all $x \in A \phi^{n} x$ belongs to $A$ for infinitely many integers $n$. It is not difficult to see that every measurable transformation which preserves a finite invariant measure $\mu$ equivalent to $m$ is recurrent. The converse statement is not in general true; for example an ergodic transformation which preserves an infinite and $\sigma$-finite measure is always recurrent yet it does not preserve a finite invariant equivalent measure. In this paper we restrict the notion of a recurrent transformation. We introduce the notion of a strongly recurrent set and define a strongly recurrent transformation. We show that a transformation $\phi$ is strongly recurrent if and only if there exists a finite invariant measure $\mu$ equivalent to $m$ (Theorem 2). This is accomplished by showing the connection between strongly recurrent sets and weakly wandering sets (Theorem 1). Weakly wandering sets were introduced in [1], and the condition that a transformation $\phi$ does not have any weakly wandering set of positive measure was further strengthened (see condition $(W)^{*}$ below). It was shown in [1] that this stronger condition was again a necessary and sufficient condition for the existence of a finite invariant measure $\mu$ equivalent to $m$. We show that a similar strengthening for a strongly recurrent transformation is false for a wide class of measure preserving transformations defined on a finite measure space (Theorem 3).

Definition. A measurable set $S$ is said to be strongly recurrent

Received July 22, 1963. 
(with respect to $\phi$ ) if the set of all integers $n$ such that $m\left(\phi^{n} S \cap S\right)>0$ is relatively dense, i.e., if there exists a positive integer $k$ such that

$$
\max _{0 \leqq i \leqq k-1} m\left(\phi^{n-i} S \cap S\right)>0
$$

for $n=0, \pm 1, \pm 2, \cdots$. This condition is obviously equivalent to the following:

$$
m\left(\bigcup_{i=0}^{k-1} \phi^{n-i} S \cap S\right)>0
$$

or

$$
m\left(\phi^{n} S \cap\left[\bigcup_{i=0}^{k-1} \phi^{i} S\right]\right)>0
$$

for $n=0, \pm 1, \pm 2, \cdots$, This last condition means that there exists a finite number of images of $S$ by the powers of $\phi$ such that any image of $S$ by any power of $\phi$ has an intersection of positive measure with at least one of them.

The transformation $\phi$ is said to be strongly recurrent if every set of positive measure is strongly recurrent. We note that the property of a transformation $\phi$ being strongly recurrent is preserved under equivalent measures.

The following notion was introduced in [1]: A measurable set $W$ is said to be weakly wandering (with respect to $\phi$ ) if there exists a sequence of integers $\left\{n_{k}: k=1,2, \cdots\right\}$ such that the sets $\phi^{n_{k}} W$, $k=1,2, \cdots$ are mutually disjoint.

Theorem 1. Let $(X, \mathscr{B}, m)$ be a finite or $\sigma$-finite measure space, and let $\phi$ be a one-to-one, nonsingular, measurable transformation of $X$ onto itself. Then the following two conditions are equivalent:

(W) $m(A)>0$ implies that there exists at most a finite number of mutually disjoint images of $A$ by the powers of $\phi$; in other words, $A$ is not weakly wandering.

(S) $m(A)>0$ implies that $A$ is strongly recurrent.

We first prove a Lemma which is by itself of some interest.

Lemma 1. Let $(X, \mathscr{B}, m)$ and $\phi$ be as in Theorem 1 , and let $A$ be a measurable set of positive measure such that

$$
\lim _{n \rightarrow \infty} \inf m\left(\phi^{n} A\right)=0 \text {. }
$$

Then given $\varepsilon$ with $0<\varepsilon<m(A)$, there exists a measurable subset $A^{\prime}$ of $A$ with $m\left(A^{\prime}\right)<\varepsilon$ such that the set $S=A-A^{\prime}$ is not strongly recurrent. 
Proof. Let $A$ be a measurable set with $m(A)=\alpha>0$ and $\lim \inf m\left(\phi^{n} A\right)=0$. Let $\varepsilon$ be a positive number with $0<\varepsilon<\alpha$. Let

$$
\varepsilon_{k}=\frac{\varepsilon}{k 2^{k}}
$$

for $k=1,2, \cdots$. Next, for each $k=1,2, \cdots$ we choose a positive integer $n_{k}$ such that

$$
m\left(\phi^{n_{k}-i} A\right)<\varepsilon_{k}
$$

for $i=0,1,2, \cdots, k-1$. This is possible since $\phi$ is nonsingular and (4) is satisfied by $A$. Let us put

$$
A^{\prime}=\bigcup_{k=1}^{\infty} \bigcup_{i=0}^{k-1} \phi^{n_{k}-i} A \cap A \text {. }
$$

Then

$$
m\left(A^{\prime}\right) \leqq \sum_{k=1}^{\infty} \sum_{i=0}^{k-1} m\left(\phi^{n_{k}-i} A\right)<\sum_{k=1}^{\infty} k \varepsilon_{k}=\varepsilon .
$$

Let $S=A-A^{\prime}$, then it is easy to see that

$$
\phi^{n_{k}-i} S \cap S \subset \phi^{n_{k}-i} A \cap\left(A-A^{\prime}\right)=\phi
$$

for $i=0,1,2, \cdots, k-1$ and $k=1,2, \cdots$. This shows that $S$ is not strongly recurrent.

Proof of Theorem 1. If a measurable set $S$ of positive measure is not strongly recurrent, then it is possible to find a measurable subset $N$ of $S$ with $m(N)=0$ such that $S^{\prime}=S-N$ is weakly wandering. This is easy, since $S$ not strongly recurrent means that for each positive integer $n_{k}$ there exists another positive integer $n_{k+1}$ such that

$$
m\left(\phi^{n_{k+1}} S \cap \bigcup_{i=0}^{n_{k}} \phi^{i} S\right)=0 .
$$

In this way we may obtain a sequence of integers $\left\{n_{k}: k=1,2, \cdots\right\}$ such that

$$
m\left(\phi^{n_{k}} S \cap \phi^{n_{j}} S\right)=m\left(S \cap \phi^{n_{k}-n_{j}} S\right)=0 \text { for } k \neq j .
$$

It follows that $S^{\prime}=S-N$ is weakly wandering, where

$$
N=\bigcup_{k=1}^{\infty} \bigcup_{j=1}^{k-1} \phi^{n_{k}-n_{j}} S \cap S
$$

and $m(N)=0$.

Conversely, let $W$ be a weakly wandering set of positive measure. 
Since the measure space is $\sigma$-finite we can find a sequence of measurable sets $\left\{A_{i}: i=1,2, \cdots\right\}$ which are mutually disjoint, such that $0<m\left(A_{i}\right)<\infty$ for $i=1,2, \cdots$ and $X=\bigcup_{i=1}^{\infty} A_{i}$. We let

$$
m^{\prime}(B)=\sum_{i=1}^{\infty} \frac{m\left(A_{i} \cap B\right)}{2^{i} m\left(A_{i}\right)} \text { for } B \in \mathscr{B} .
$$

It follows that $m^{\prime}$ and $m$ are equivalent. Since $\phi^{n_{k}} W, k=1,2, \cdots$ are mutually disjoint and $m^{\prime}(X)<\infty$ it follows that $\lim \inf m^{\prime}\left(\phi^{n} W\right)=$ 0 . Thus, whether $m$ is finite or $\sigma$-finite, the set $W$ satisfies (4) with $m$ replaced by the equivalent and finite measure $m^{\prime}$. By applying Lemma 1 we obtain a measurable subset $S$ of $W$ such that $m^{\prime}(S)>0$ and $S$ is not strongly recurrent. Since $m$ and $m^{\prime}$ are equivalent, this proves the theorem.

Theorem 2. Let $(X, \mathscr{B}, m)$ and $\phi$ be as in Theorem 1. Then condition $(S)$ is equivalent to the existence of a finite invariant measure $\mu$ equivalent to $m$.

Proof. Theorem 2 is an immediate consequence of Theorem 1 above and Theorem 1 of [1], where it was shown that condition $(W)$ is equivalent to the existence of a finite invariant measure $\mu$ equivalent to $m$.

In [1] it was further shown that the following condition:

$(\mathrm{W})^{*}$ Given $\varepsilon>0$, there exists a positive integer $N$ such that $m(A) \geqq \varepsilon$ implies that there exists at most $N$ mutually disjoint images of $A$ by the powers of $T$,

is again a necessary and sufficient condition for the existence of a finite invariant measure $\mu$ equivalent to $m$ (see condition $(V)^{*}$, $\S 3$ of [1]).

Condition $(W)^{*}$ is in appearance a stronger condition than condition (W). We note that in condition (W)* the positive integer $N$ depends on $\varepsilon$ only and not on the measurable set $A$. However, it turns out that these two conditions are equivalent to each other and are in turn necessary and sufficient conditions for the existence of a finite invariant measure $\mu$ equivalent to $m$ (see Theorem 1 of [1]). By analogy, we may attempt to strengthen condition (S) in the following manner:

$(\mathrm{S})^{*}$ Given $\varepsilon>0$, there exists a positive integer $N$ such that $m(A) \geqq \varepsilon$ implies

$$
m\left(\phi^{n} A \cap \bigcup_{i=0}^{N-1} \phi^{i} A\right)>0 \text { for } n=0, \pm 1, \pm 2, \cdots
$$

We show that condition (S)* is not a necessary condition for the 
existence of a finite invariant measure $\mu$ equivalent to $m$. In fact, we shall show that for any ergodic measure preserving transformation $\phi$ defined on a finite measure space $(X, \mathscr{B}, \mu)$ condition $(\mathrm{S})^{*}$ is not satisfied.

We say that a transformation $\phi$ is ergodic if $\phi A=A$ implies $m(A)=0$ or $m(X-A)=0$.

Lemma 2. Let $(X, \mathscr{B}, \mu$,$) be a finite or \sigma$-finite measure space, and let $\phi$ be an ergodic measure preserving transformation defined on it. Then given $\varepsilon>0$ and a positive integer $N>0$, there exists a measurable set $C$ with $\mu(C) \leqq \varepsilon$ such that

$$
X-C=\bigcup_{i=0}^{N-1} \phi^{i} E
$$

for some measurable set $E$ where $E, \phi E, \cdots, \phi^{N-1} E$ are mutually disjoint.

Proof. Given $\varepsilon>0$ and an integer $N>0$, let $F$ be any measurable set with $0<\mu(F) \leqq \varepsilon / N$. Let

$$
\begin{aligned}
& F_{0}=F \\
& F_{1}=\phi^{-1} F-F_{0} \\
& F_{2}=\phi^{-2} F-F_{0} \cup F_{1}
\end{aligned}
$$

and in general

$$
F_{n}=\phi^{-n} F-\bigcup_{j=0}^{n-1} F_{j} \text { for } n=1,2, \cdots .
$$

It follows that $F_{n}, n=0,1,2, \cdots$ are mutually disjoint, and furthermore;

$$
\begin{array}{ll}
\phi^{k} F_{n} \subset F_{n-k} & \text { for } k=0, \cdots, n \\
& \text { and } n=0,1,2, \cdots .
\end{array}
$$

We let

$$
E_{i}=F_{i N}=\phi^{-i N} F-\bigcup_{j=0}^{i N-1} F_{j}=\phi^{-i N} F-\bigcup_{j=0}^{i N-1} \phi^{-j} F
$$

then

$$
\phi^{k} E_{i} \subset F_{i N-k} \text { for } k=0,1, \cdots, i N \text {; and } i=1,2, \cdots
$$

which implies that the sets

$$
\phi^{k} E_{i} \quad \text { for } k=0,1, \cdots, i N \text {; and } j=1,2, \cdots
$$

are mutually disjoint. 
Next we let

$$
E=\bigcup_{i=1}^{\infty} E_{i}
$$

and

$$
C=X-\bigcup_{k=0}^{N-1} \phi^{k} E
$$

It follows from (5) that $E, \phi E, \cdots, \phi^{N-1} E$ are mutually disjoint, and

$$
\mu(C)=\mu\left(X-\bigcup_{k=0}^{N-1} \phi^{k} E\right) \leqq N \mu(F) \leqq \varepsilon .
$$

THEOREM 3. Let $\phi$ be on ergodic measure preserving transformation defined on a finite measure space $(X, \mathscr{B}, \mu)$ with $\mu(X)=1$. Then condition $(S)^{*}$ is not satisfied.

Proof. Let $\varepsilon=1 /(q+1)$ for some positive integer $q>3$. Let $k>1$ be an arbitrary positive integer. We show that there exists a measurable set $A$ with $\mu(A) \geqq \varepsilon$ and

$$
\mu\left(\phi^{n_{k}} A \cap \bigcup_{i=0}^{k-1} \phi^{i} A\right)=0
$$

for some integer $n_{k}>k$. Let us put $N=q k$. Then by Lemma 2 there exists a measurable set $E$ with $E, \phi E, \cdots, \phi^{N-1} E$ mutually disjoint and

$$
\mu\left(X-\bigcup_{k=0}^{N-1} \phi^{k} E\right) \leqq \varepsilon=\frac{1}{q+1} .
$$

Since $\mu(X)=1$, this implies $1-N \mu(E) \leqq \varepsilon$ or $\mu(E) \geqq(1-\varepsilon) / N$. Let

$$
A=\bigcup_{i=0}^{k-1} \phi^{i} E
$$

Since $k=N / q$ we have

$$
\mu(A)=k \mu(E) \geqq \frac{N}{q} \frac{(1-\varepsilon)}{N}=\frac{1-\frac{1}{q+1}}{q}=\frac{1}{q+1}=\varepsilon
$$

and

$$
\mu\left(\phi^{n_{k}} A \cap \bigcup_{i=0}^{k-1} \phi^{i} A\right)=\mu\left(\bigcup_{i=n_{k}}^{n_{k}+k_{k}-1} \phi^{i} E \cap \bigcup_{i=0}^{2 k-2} \phi^{i} E\right)=0
$$

for some $n_{k}$ where $2 k<n_{k}<(q-1) k=N-k$. 
This shows that condition (S)* is not satisfied since $\varepsilon$ is fixed, $k$ is arbitrary, and $n_{k}>k$.

\section{REFERENCE}

1. A. Hajian and S. Kakutani, Weakly wandering sets and invariant measures, Transactions A. M. S. 110 (1964), 136-151.

CORNELL UNIVERSITY 



\title{
PACIFIC JOURNAL OF MATHEMATICS
}

\author{
EDITORS
}

\author{
Robert Osserman \\ Stanford University \\ Stanford, California \\ M. G. Arsove \\ University of Washington \\ Seattle 5, Washington
}

\author{
J. Dugundji \\ University of Southern California \\ Los Angeles 7, California \\ Lowell J. Paige \\ University of California \\ Los Angeles 24, California
}

\section{ASSOCIATE EDITORS}
E. F. BECKENBACH
B. H. NEUMANN
F. WOLF
K. YOSHIDA

\section{SUPPORTING INSTITUTIONS}

\author{
UNIVERSITY OF BRITISH COLUMBIA \\ CALIFORNIA INSTITUTE OF TECHNOLOGY \\ UNIVERSITY OF CALIFORNIA \\ MONTANA STATE UNIVERSITY \\ UNIVERSITY OF NEVADA \\ NEW MEXICO STATE UNIVERSITY \\ OREGON STATE UNIVERSITY \\ UNIVERSITY OF OREGON \\ OSAKA UNIVERSITY \\ UNIVERSITY OF SOUTHERN CALIFORNIA
}

\author{
STANFORD UNIVERSITY \\ UNIVERSITY OF TOKYO \\ UNIVERSITY OF UTAH \\ WASHINGTON STATE UNIVERSITY \\ UNIVERSITY OF WASHINGTON \\ AMERICAN MATHEMATICAL SOCIETY \\ CALIFORNIA RESEARCH CORPORATION \\ SPACE TECHNOLOGY LABORATORIES \\ NAVAL ORDNANCE TEST STATION
}

Mathematical papers intended for publication in the Pacific Journal of Mathematics should by typewritten (double spaced), and on submission, must be accompanied by a separate author's résumé. Manuscripts may be sent to any one of the four editors. All other communications to the editors should be addressed to the managing editor, L. J. Paige at the University of California, Los Angeles 24, California.

50 reprints per author of each article are furnished free of charge; additional copies may be obtained at cost in multiples of 50 .

The Pacific Journal of Mathematics is published quarterly, in March, June, September, and December. Effective with Volume 13 the price per volume (4 numbers) is $\$ 18.00$; single issues, $\$ 5.00$. Special price for current issues to individual faculty members of supporting institutions and to individual members of the American Mathematical Society: $\$ 8.00$ per volume; single issues $\$ 2.50$. Back numbers are available.

Subscriptions, orders for back numbers, and changes of address should be sent to Pacific Journal of Mathematics, 103 Highland Boulevard, Berkeley 8, California.

Printed at Kokusai Bunken Insatsusha (International Academic Printing Co., Ltd.), No. 6, 2-chome, Fujimi-cho, Chiyoda-ku, Tokyo, Japan.

PUBLISHED BY PACIFIC JOURNAL OF MATHEMATICS, A NON-PROFIT CORPORATION

The Supporting Institutions listed above contribute to the cost of publication of this Journal, but they are not owners or publishers and have no responsibility for its content or policies. 


\section{Pacific Journal of Mathematics}

\section{Vol. 14, No. $2 \quad$ June, 1964}

Tom M. (Mike) Apostol and Herbert S. Zuckerman, On the functional equation $F(m n) F((m, n))=F(m) F(n) f((m, n)) \ldots \ldots \ldots \ldots \ldots \ldots \ldots \ldots \ldots \ldots \ldots$

Reinhold Baer, Irreducible groups of automorphisms of abelian groups . . . . . . . 385

Herbert Stanley Bear, Jr., An abstract potential theory with continuous kernel . . . . 407

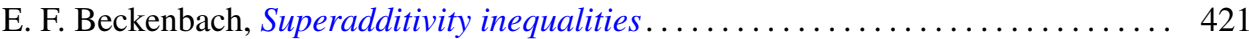

R. H. Bing, The simple connectivity of the sum of two disks . . . . . . . . . . . 439

Herbert Busemann, Length-preserving maps ...................... 457

Heron S. Collins, Characterizations of convolution semigroups of measures . . . . . . 479

Paul F. Conrad, The relationship between the radical of a lattice-ordered group and complete distributivity............................ 493

P. H. Doyle, III, A sufficient condition that an arc in $S^{n}$ be cellular . . . . . . . . . 501

Carl Clifton Faith and Yuzo Utumi, Intrinsic extensions of rings . . . . . . . . . . 505

Watson Bryan Fulks, An approximate Gauss mean value theorem . . . . . . . . . . 513

Arshag Berge Hajian, Strongly recurrent transformations . . . . . . . . . . . . . 517

Morisuke Hasumi and T. P. Srinivasan, Doubly invariant subspaces. II . . . . . . . 525

Lowell A. Hinrichs, Ivan Niven and Charles L. Vanden Eynden, Fields defined by

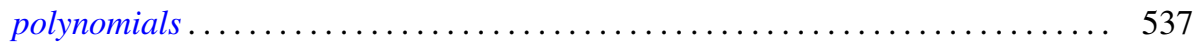

Walter Ball Laffer, I and Henry B. Mann, Decomposition of sets of group

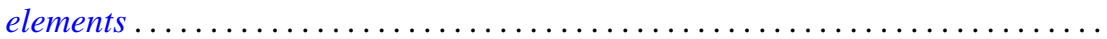

John Albert Lindberg, Jr., Algebraic extensions of commutative Banach

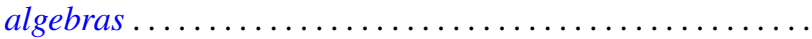

W. Ljunggren, On the Diophantine equation $C x^{2}+D=y^{n} \ldots$

M. Donald MacLaren, Atomic orthocomplemented lattices ....

Moshe Marcus, Transformations of domains in the plane and applications in the

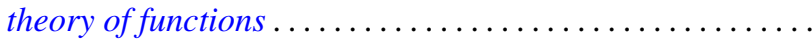

Philip Miles, $B^{*}$ algebra unit ball extremal points . .................. 627

W. F. Newns, On the difference and sum of a basic set of polynomials . . . . . . . 639

Barbara Osofsky, Rings all of whose finitely generated modules are injective ...... 645

Calvin R. Putnam, Toeplitz, matrices and invertibility of Hankel matrices . . . . . . . 651

Shoichiro Sakai, Weakly compact operators on operator algebras . . . . . . . . . 659

James E. Simpson, Nilpotency and spectral operators . . . . . . . . . . . . . 665

Walter Laws Smith, On the elementary renewal theorem for non-identically

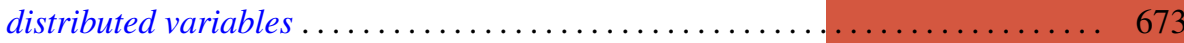

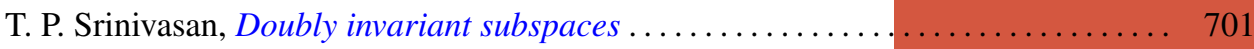

J. Roger Teller, On the extensions of lattice-ordered groups . . . . . . . . . . . . 709

Robert Charles Thompson, Unimodular group matrices with rational integers as

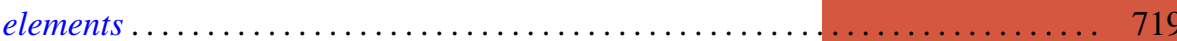

J. L. Walsh and Ambikeshwar Sharma, Least squares and interpolation in roots of unity

Charles Edward Watts, A Jordan-Hölder theorem .................... 731

Kung-Wei Yang, On some finite groups and their cohomology .............. 735

Adil Mohamed Yaqub, On the ring-logic character of certain rings ............ 741

Paul Ruel Young, A note on pseudo-creative sets and cylinders 